\title{
Experiences of using a single post-contrast CT scan of the chest after biphasic contrast injection
}

\author{
P C Pretorius, FCRad (Diag) SA \\ Drs Visser, Erasmus, Vawda \& Partners, Port Elizabeth
}

Corresponding author: P Pretorius (ppret@telkomsa.net)

Computed tomographic (CT) chest investigations can be enhanced; in many cases, the arterial phase of a post-contrast arterial and delay (60 seconds) study can be omitted when planning the contrast injection and scanning technique carefully. A biphasic contrast injection was used before starting a single 60 -second post-contrast scan.

S Afr J Rad 2012;16(2):56-60.

\section{Introduction}

While experimenting with single post-contrast computed tomographic (CT) scans of the urinary tract after a triphasic contrast injection, ${ }^{1} \mathrm{I}$ considered whether a similar technique could be used to cut down on unnecessary scan series in our patients referred for chest CT scans. A precontrast scan and then a biphasic contrast injection followed by a single scan of the thorax was therefore considered and implemented to test the reliability and quality of this technique.

Protocols for chest CT surveys for mediastinal pathology, lung masses and 'rule out pathology' chest scans traditionally dictate a postcontrast scan beginning $60 \mathrm{~s}$ after initiating an intravenous contrast injection. In numerous practices, $100-120 \mathrm{cc}$ of contrast is traditionally used. This process usually results in adequate contrast throughout the vascular structures in the mediastinum and lungs and enhancement of pathologic masses or lymph nodes.

When there are clinical indications of vascular pathology (i.e. aortic or pulmonary arteries and their respective branch vessels), it is imperative to add a dedicated arterial phase scan before the $60 \mathrm{~s}$ scan series. If there are no clinical indications to include an arterial phase, a single 60 s post-contrast scan only should be performed.
Unfortunately, clinical information from referring physicians is not always clear and, even when clear, many radiologists feel insecure without including an arterial series. We felt that this biphasic injection followed by a single post-contrast scan technique improved the diagnostic information and also alleviated the insecurity of excluding the arterial series when performing chest CT scans for non-vascular indications.

After scanning a few test cases using a single post-contrast series after a biphasic contrast injection, we were pleasantly surprised with the results, and we have now introduced this technique in most of our practice, when a 'general rule out' or 'mass lesion characterisation' scan is requested. This requires radiologists to shift their comfort zones, to rely on a single post-contrast scan replacing either a post-contrast arterial, $60 \mathrm{~s}$, or both arterial and $60 \mathrm{~s} \mathrm{scans.}$

I have included a pictorial representation of a variety of pathologies while using the newer technique, to share our experiences over a wide range of pathologies, which will assist our colleagues in making this shift of technique with more confidence, and will hopefully address some of the queries that may be raised.

\section{Injection plan}

1. $80 \mathrm{cc}$ contrast @ $3 \mathrm{cc} / \mathrm{s}$ (27 s) - contributes to 'venous phase' of the scan

2. $20 \mathrm{cc}$ saline @ $3 \mathrm{cc} / \mathrm{s}(7 \mathrm{~s})$ - pushes contrast into system.

3. Delay (11 s)

4. $40 \mathrm{cc}$ contrast @ $2.5 \mathrm{cc} / \mathrm{s}$ (16 s) - contributes to the 'arterial phase' of the scan

5. Followed by $20 \mathrm{cc}$ saline @ $2.5 \mathrm{cc} / \mathrm{s}$

6. The scan is started at $60 \mathrm{~s}$, which is $15 \mathrm{~s}$ into the arterial phase of the injection, $1 \mathrm{~s}$ before the end of the injection. Most scanners require a $4-5 \mathrm{~s}$ delay from the time the scan is initiated until the first 'cuts' are taken - see comments in the discussion below.

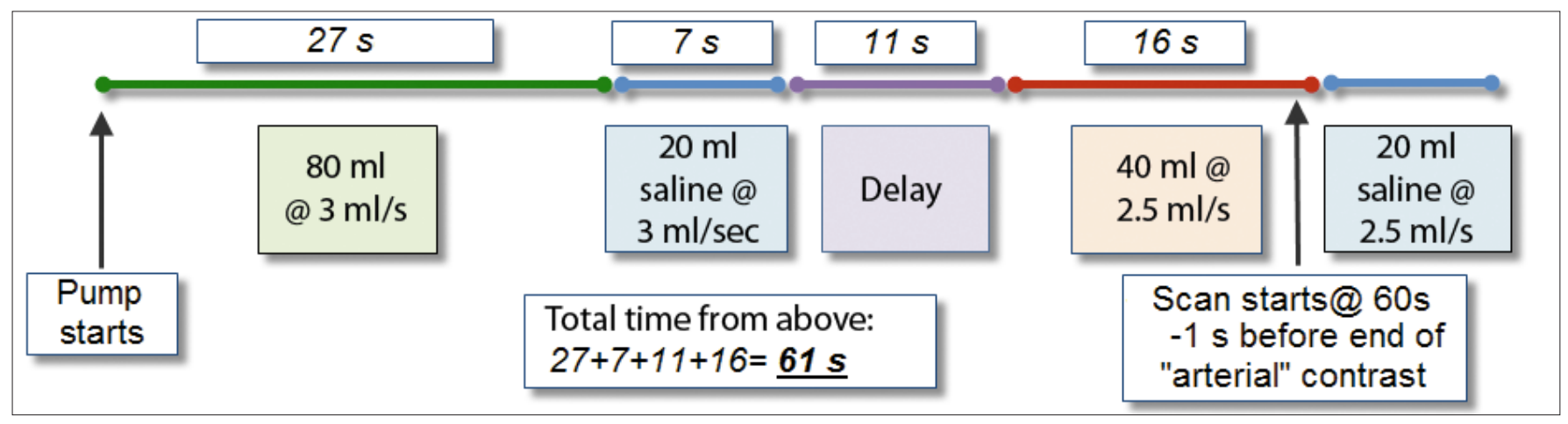

Fig. 1. Injection plan. 


\section{PICTORIAL INTERLUDE}

Case studies

Normal arterial phase scan (Fig. 2)

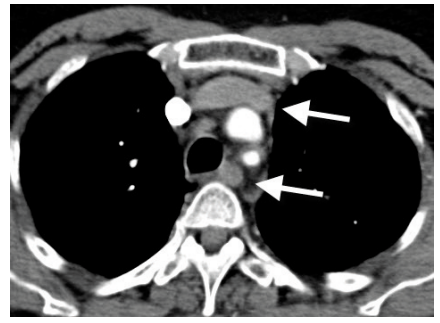

$2 a$

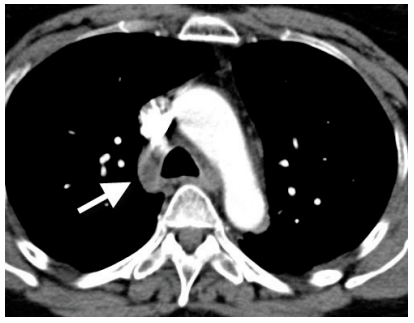

$2 b$ and unenhanced lymph nodes posteriorly. In Fig. $2 b$, the white arrow indicates the unenhanced azygos vein.

Arterial phase-contrast scans of the chest show good contrast enhancement of pulmonary arteries and veins, heart chambers, the aorta and its branch vessels. There is good differentiation of contrast between the central venous and arterial structures (dense enhancement v. no enhancement). The returning systemic veins contralateral to the side injected, from the head and neck, from infradiaphragmatic veins (especially the azygos vein) and any mediastinal and hilar masses and lymph nodes and lung pathology are not enhanced at this early postcontrast phase. Consequently, there is often difficulty in differentiating the unenhanced dilated or anomalous venous structures from the unenhanced lymph nodes and masses in the chest.

Normal 60 s post-contrast scan (Fig. 3)

The contrast enhancement of the vascular structures, mass lesions and lymph nodes within the chest is usually adequate, but differentiation between central arterial and venous structures and returning systemic venous structures relies on anatomical identification, as the contrast density of these structures is usually very similar. The contrast in the pulmonary arteries and branches is often mediocre, and incidental pulmonary arterial emboli may not be well seen.

Post biphasic contrast injection scan (Fig. 4)

After a biphasic contrast injection, there is dense enhancement of central pulmonary arteries and veins and the aorta and its branches. Returning systemic veins also show enhancement, but less dense than the central vessels, allowing further clarity to confirm the anatomical differentiation. Lung and mediastinal masses and lymph nodes will also enhance, usually to a lesser degree than systemic veins, and will show their enhancement patterns.

\section{Lung carcinoma after biphasic contrast injection} (Fig. 5)

Despite the scan beginning at $60 \mathrm{~s}$, there is dense enhancement of the pulmonary arteries, veins and aorta, without compromising enhancement and characterisation of the lung mass and lymph nodes.

\section{Mycetoma after biphasic contrast injection (Fig. 6)}

It is interesting to compare this case with the preceding lung carcinoma case (Fig. 5), where there was enhancement of the mass and the typical peripheral enhancement of infiltrated lymph nodes, unlike the nonenhancement of this mass and the uniform node enhancement.

\section{Lymphoma after biphasic contrast injection (Fig. 7)} Having dense contrast in all the vascular structures, with enhancement of the mass, all on one post-contrast series, helped to 'unpack' all the pathological processes going on in this case, which initially appeared quite complex because of the gross changes.

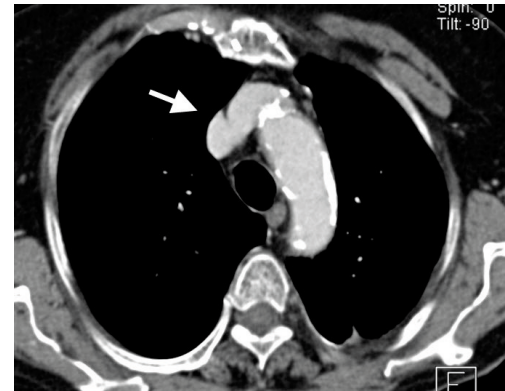

$3 a$

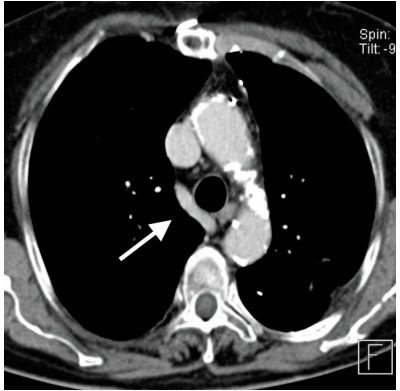

$3 b$

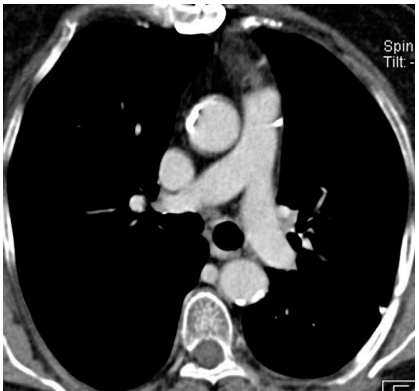

$3 c$

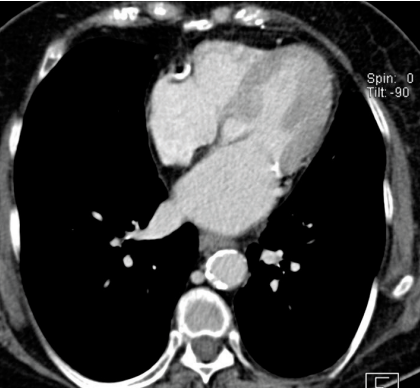

$3 d$

Fig. 3. Scan of the chest $60 \mathrm{~s}$ after contrast injection. Arrows in $3 a$ and $3 b$ point to the left brachiocephalic vein and azygos vein, respectively.

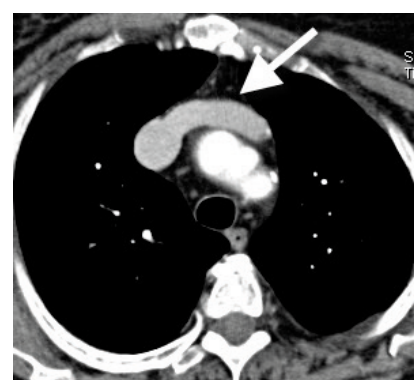

$4 a$

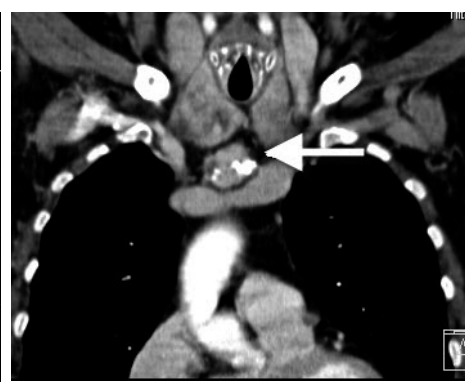

$4 c$

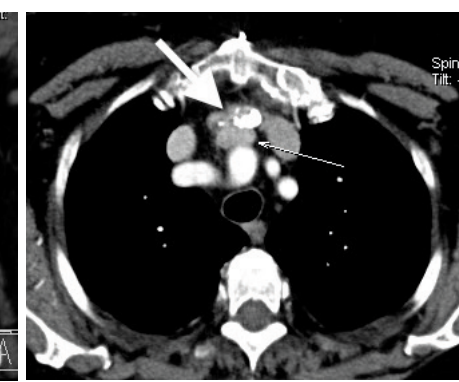

$4 d$

Fig. 4. Biphasic contrast injection single post-contrast injection series. The white arrow in $4 a$ and $4 b$ shows contrast-enhanced left brachiocephalic and azygos veins. The white arrow in Figs $4 c$ and $4 d$ show an enhancing ectopic thyroid nodule, with calcification, abutting on the enhanced left brachiocephalic vein. 


\section{PICTORIAL INTERLUDE}

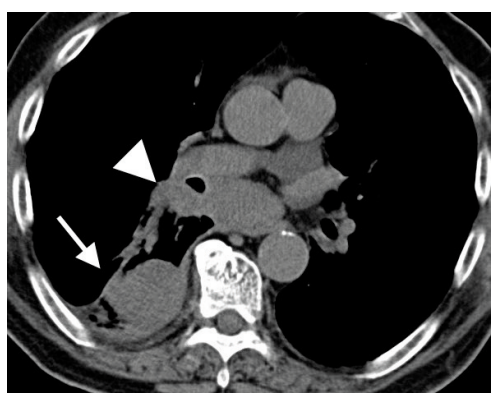

$5 a$

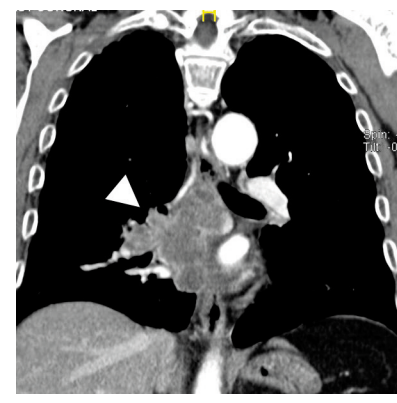

$5 c$

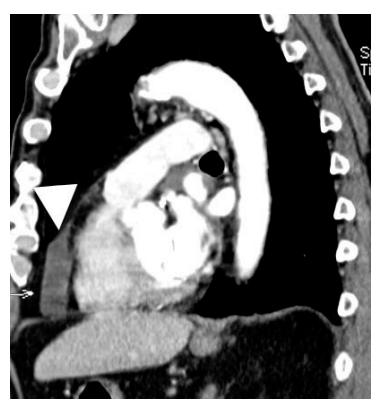

$5 d$

nodes show typical peripheral enhancement on the post-contrast series (arrowheads in $5 b, 5 c$ and $5 d$ ).

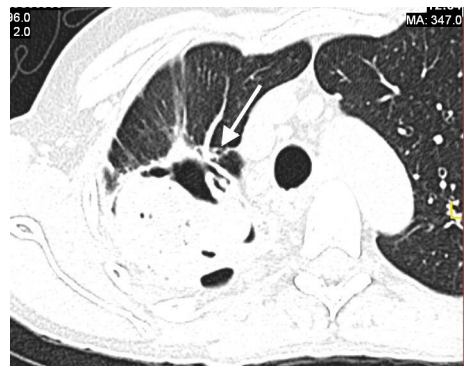

$6 a$ inflammatory uniformly enhancing lymph nodes in the adjacent mediastinum - arrow in $6 \mathrm{~d}$.

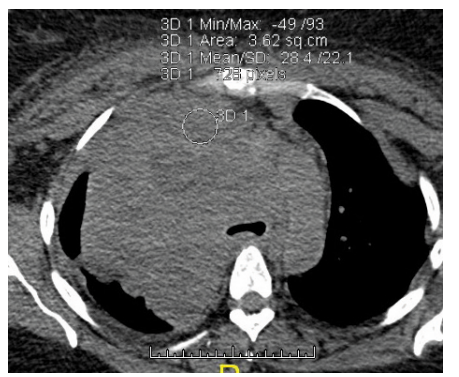

$7 a$

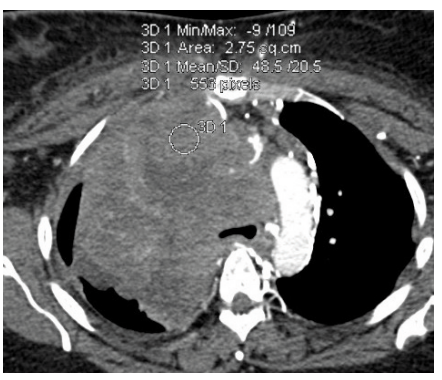

76

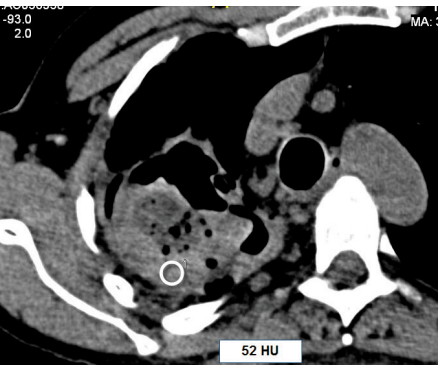

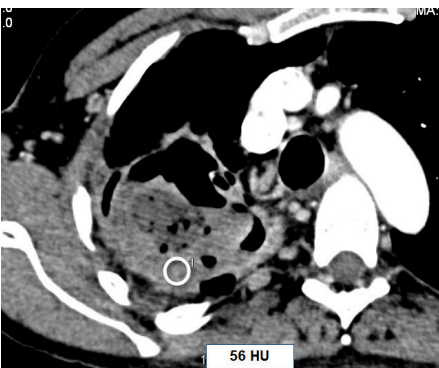

$6 c$

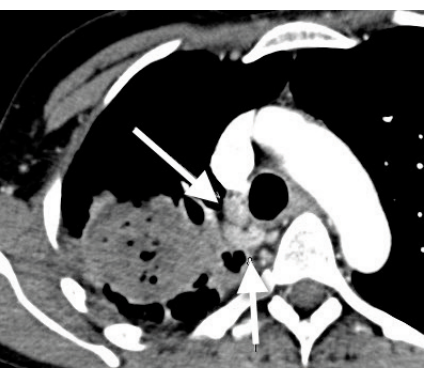

$6 d$

the mass. Infiltrated lymph 


\section{PICTORIAL INTERLUDE}

Opportunistic infection after biphasic contrast injection (Fig. 11)

The pulmonary arterial emboli might not have been as clearly seen on a single standard post-contrast $60 \mathrm{~s} \mathrm{scan}$, and the splenic micro-abscesses might also have been missed on a standard post-arterial scan. These were all seen on the single post-contrast series.
Atelectasis after biphasic contrast injection (Fig. 12)

The collapsed lung is enhanced, with dense enhancement of vascular structures.

\section{Discussion}

The contrast load traditionally used for a survey chest CT is $100-120 \mathrm{cc}$, to gain adequate contrast density in vascular structures in the chest. A 'rule

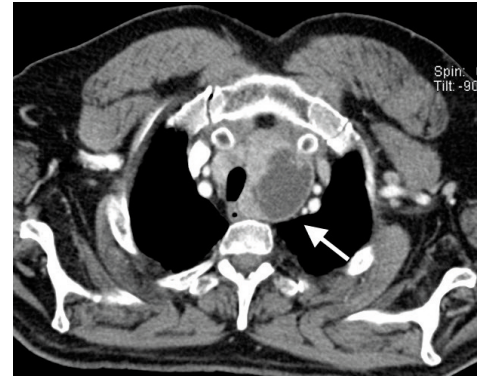

$8 a$

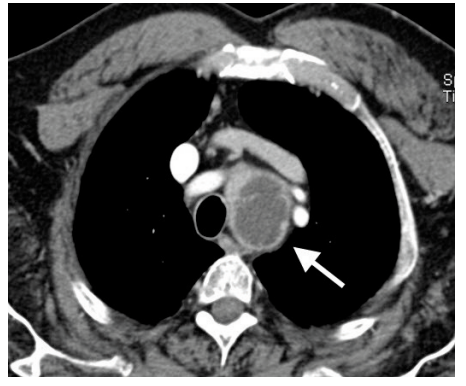

$8 b$

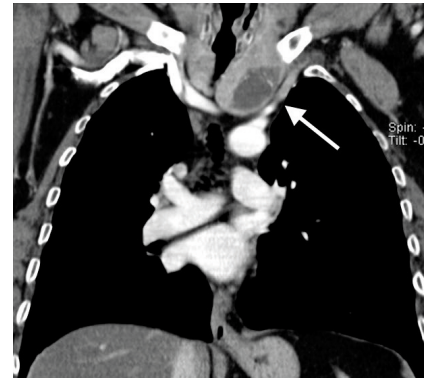

$8 c$

Fig. 8. Retrosternal extension of a goitre (arrow), with cystic change in left lobe. Clear separation and differentiation from adjacent aortic arch branches bilaterally and the enhanced left brachiocephalic vein anteriorly noted in $8 b$.

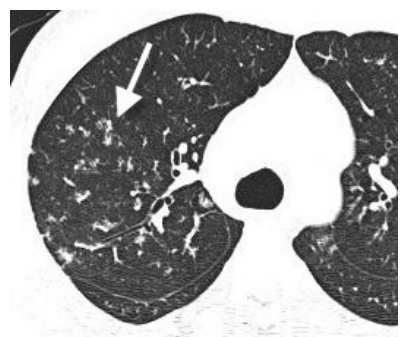

$9 a$

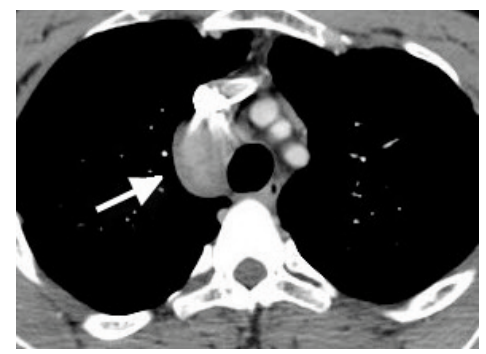

96

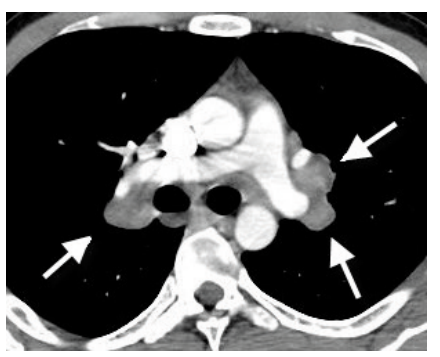

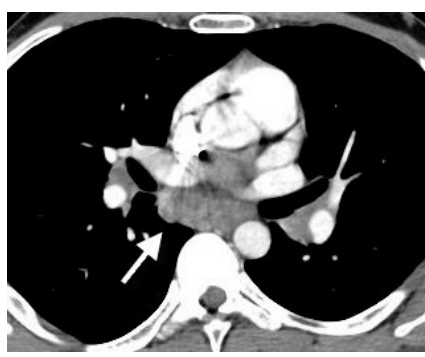

Fig. 9. Sarcoid infiltration of lung (arrow in 9a), with enlarged uniformly enhancing mediastinal and hilar lymph nodes (arrows in $9 b$ - d).

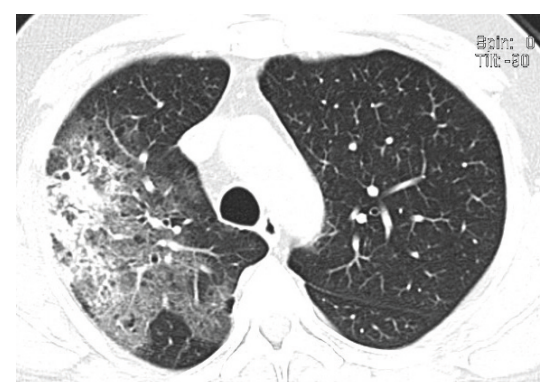

$10 a$

Fig. 10. Interstitial changes in the right lung. There is mediastinal lymphadenopathy (arrow in 10b) differentiated from the adjacent enhanced azygos vein (arrowhead in 10b).

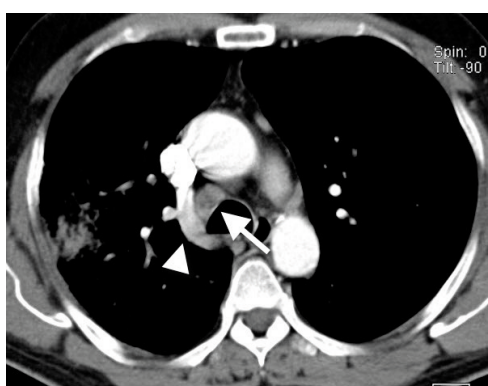

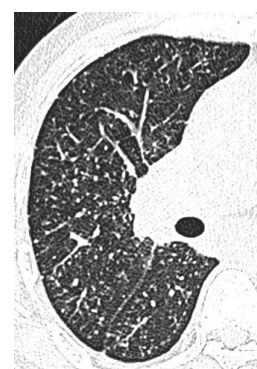

$\$$

$11 a$

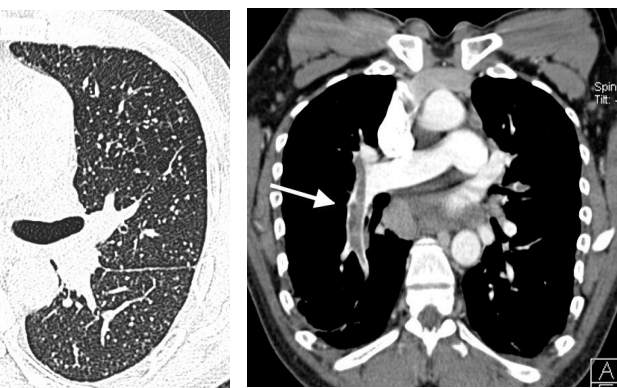

116

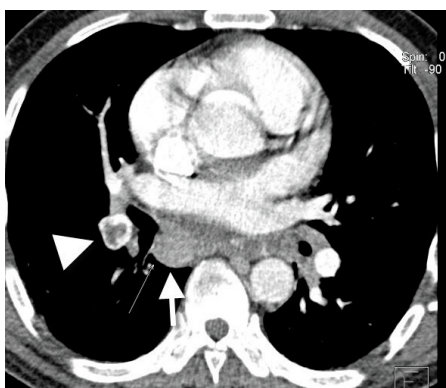

$11 c$

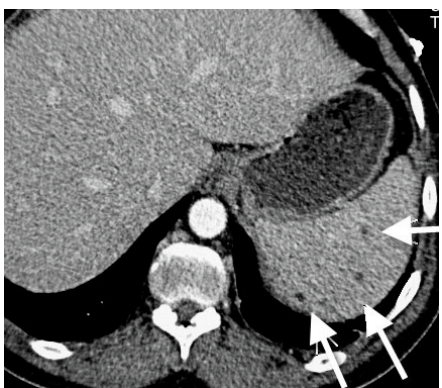

$11 d$

portunistic nodular lung infection in immunocompromised patient. Unsuspected pulmonary arterial emboli noted on the right (arrow in $11 b$ and arrowhead in 11c). Lymph node enlargement noted (arrow in 11c). Micro-abscesses also identified in spleen (arrows in 11d) at the inferior range of the scan. 


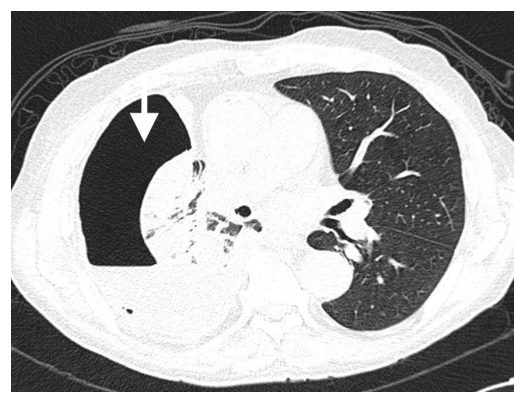

$12 a$

Fig. 12. Right pneumothorax (arrow in 12a) with atelectasis of underlying lung (arrow in 12b) and pleural fluid.

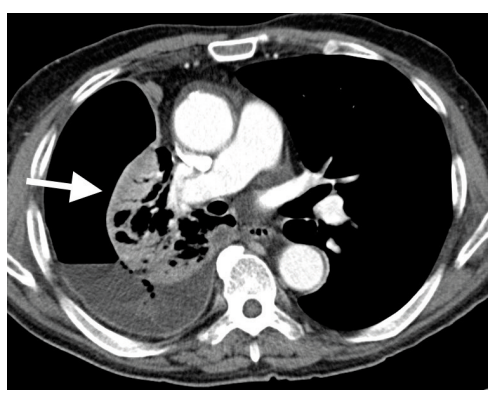

$12 b$ of thumb' is that, from the time the contrast injection into an antecubital vein begins, it takes $9 \mathrm{~s}$ to reach the heart, by $12 \mathrm{~s}$ it is through the lungs back to the heart, and by $15 \mathrm{~s}$ has reached the descending thoracic aorta.

Contrast injection rates vary between $2-5 \mathrm{cc} / \mathrm{s}$, depending on the sequence chosen. A contrast injection of $100 \mathrm{cc}$ at $4 \mathrm{cc} / \mathrm{s}$. will ensure a $25 \mathrm{~s}$ bolus of contrast. Since modern multislice scanners can scan the whole thorax in $10-16 \mathrm{~s}$ and the resolution is so high, it is conceivable that, if one could get the timing perfect in every patient, one could theoretically perform a good arterial phase diagnostic scan of the chest with only $16 \mathrm{~s} \times 2.5 \mathrm{cc} / \mathrm{s}=40 \mathrm{cc}$ of contrast.

Naturally, there are numerous variable factors in patients such as body morphology, cardiac function, degree of valsalva with breath hold, etc. 'Perfect timing' of the scan in every case is consequently not feasible but, as can be seen from the above description, a theoretical $60-80 \mathrm{cc}$ of contrast $(100-120 \mathrm{cc}$ minus $40 \mathrm{cc})$ is non-contributory to the diagnostic information, if one were looking purely at the arterial filling of vessels. This 'extra' contrast can be used to enhance the contrast appearances in the other vessels by splitting the bolus delivery.

Using the above logic, we designed an injection sequence to try to maximise the benefit of this 'non-contributory' portion of contrast that we inject. The logic is as follows:

- The initial $80 \mathrm{cc}$ starting $60 \mathrm{~s}$ before the scan allows adequate contrast filling of the returning systemic veins, particularly the brachiocephalic and azygos veins. The injection rate is kept similar to that of a 'traditional' chest survey CT scan. This contrast is 'pushed' by a saline chaser injection at the same rate, followed by a calculated delay.

- A second contrast injection begins $15 \mathrm{~s}$ before the CT scan starts. This will enhance the pulmonary arterial and pulmonary venous vessels and the aortic vessels in the chest. This timing allows the end of the second phase bolus of contrast to densely enhance the central vessels (pulmonary veins and arteries), while the lead portion of this second bolus will have reached the descending aorta in almost all patients.

- In fit patients, this timing is invariably adequate.

- In patients with known poor cardiac function, the contrast passage may be a bit slower. In these cases, one may start the scan a little later - possibly at $65 \mathrm{~s}$ This is still usually adequate as the contrast bolus tends to 'stretch out' i.e. a tight bolus injected into a peripheral vein over $15 \mathrm{~s}$. will have spread out to possibly over $20 \mathrm{~s}$ by the time it has gone through the heart, lungs and heart again, before entering the aorta.

- The $60 \mathrm{~s}$ initiation of the scan after the beginning of the contrast injection, means that the scan effectively starts at $64 \mathrm{~s}$, because of the built-in delay for a scan to initiate. This is $3 \mathrm{~s}$ after completion of the contrast injection in the arm. I have found that this timing is adequate (as the contrast bolus still has to travel up the arm and through the heart and lungs and into the aorta) and the dense arterial phase of contrast in the vessels from the superior vena cava, through the heart, pulmonary arteries and veins and aorta is captured regularly with this timing.

- A final saline chaser injection pushes the contrast towards the chest.

This routine gave the following advantages:

- adequate enhancement of returning systemic veins, to help differentiate them from mediastinal and hilar masses and lymph nodes

- relatively dense contrast enhancement of pulmonary arteries, veins and thoracic aorta and its branches, which allowed clear differentiation between systemic returning veins and mediastinal and hilar masses and lymph nodes

- enhancement of masses and lymph nodes allowed their characterisation

- all the post-contrast information was seen on one set of images, negating the need to run both an arterial and a $60 \mathrm{~s}$ post-contrast series ( 2 series). This made interpretation quicker and in many cases easier (see case of lymphoma above - Fig. 7).

In my opinion, the information gained is increased when compared with a single 60 s post-contrast scan.

Radiation exposure is reduced by omitting the arterial phase scan in practices where both an arterial and $60 \mathrm{~s}$ post-contrast are run for those cases where the arterial series is not indicated, but is added for the radiologist's comfort.

Other advantages from reducing unnecessary series of post-contrast CT scans have been discussed in a previous article using a single postcontrast CT scan of the urinary tract after triphasic contrast injection. ${ }^{1}$ These advantages include:

- more efficient reading of the scans owing to more information presented on a single post-contrast series

- ease of viewing of a single scan post-contrast series for the referring clinician

- reduction in radiation to the patient from unnecessary extra scan series

- reduced wear and tear on scanner hardware

- reduction in the number of images needing electronic archiving.

It must again be emphasised that this technique is used to replace chest CT scans where the clinical information dictates a pre-contrast and a single post-contrast scan at $60 \mathrm{~s}$ and should not to be used when a dedicated arterial series is indicated.

1. Pretorius PC. Experiences of using a single post-contrast $\mathrm{CT}$ scan of the urinary tract after triphasic contrast injection. SA Journal of Radiology 2011;15(4):140-145. 